\title{
The influence of cyclic loading conditions on the viscoelastic properties of filled rubber
}

\author{
P. Garnier ${ }^{\mathrm{a}, \mathrm{b}, \mathrm{c}}$, J.-B. Le Cam ${ }^{\mathrm{b}, *}$, M. Grédiac ${ }^{\mathrm{a}}$ \\ ${ }^{a}$ Université Blaise Pascal-Institut Français de Mécanique Avancée, Laboratoire de Mécanique et Ingénieries, BP 265, 63175 Clermont-Ferrand, France \\ ${ }^{\mathrm{b}}$ Université de Rennes 1, LARMAUR, ERL CNRS 6274, Campus de Beaulieu, 35042 Rennes Cedex, France \\ ' $P C M, 1$ Rue René Moineau, F-49000 Champtocé sur Loire, France
}

This study deals with the effect of cyclic loading on the viscoelastic properties of filled nitrile rubbers. Classic strain amplitude sweeps were first carried out at two different temperatures (ambient and $80^{\circ} \mathrm{C}$ ) on both filled and unfilled nitrile rubber specimens in order to observe the influence of the fillers, temperature and loading conditions on the Payne effect. Some specimens were then subjected to a high number of cycles to study the variations in the viscoelastic properties and the sensitivity of the Payne effect to cyclic loading tests at several given strain amplitudes. It appears that the viscoelastic properties of rubber significantly evolve during the cyclic tests, which raises the question of the concept of cyclic stabilized behaviour.

\section{Introduction}

Rubber components are widely used in various fields of engineering, such as the automotive industry and aeronautics, due to their high elasticity and high damping properties. The optimal design of such components must take into account the mechanical properties of rubber (see for instance Bergström and Boyce, 2000; Le Cam and Toussaint, 2009; Palmieri et al., 2009; Machado et al., 2010; Diani et al., 2004), including viscoelasticity. Classically, the viscoelastic properties of filled rubber are studied using dynamic mechanical analysis (DMA) or dynamic mechanical and thermal analysis (DMTA). This type of analysis provides the storage modulus $E^{\prime}$, the viscous modulus $E^{\prime \prime}$, and the loss factor $\tan \delta$ for a given double strain amplitude (DSA, defined as the strain range). In filled rubber materials, a decrease in the storage modulus is generally observed if the amplitude of the cyclic strain increases. This phenomenon, called the Payne effect (Payne, 1962) or

\footnotetext{
* Corresponding author.

E-mail address: jean-benoit.lecam@univ-rennes1.fr (J.-B. Le Cam).
}

the Fletcher-Gent effect (Fletcher and Gent, 1953), is not observed in unfilled rubber. Several studies have investigated the effect of degradation processes, such as irradiation or temperature (Celina et al., 1998; Delor-Jestina et al., 2000), on the mechanical properties of rubbers. Nevertheless, the effect of cyclic loading on viscoelastic properties has not been studied in the Payne effect strain domain. This lack of information is quite surprising, since rubber parts are subjected to such mechanical cyclic loading in many industrial applications.

This paper aims to investigate variations in the viscoelastic properties of filled nitrile rubber under cyclic loading, typically up to $10^{6}$ cycles. These mechanical cycles were applied under compression. This distinguishes all the more singularly the present study, given that the viscoelastic properties of rubber are classically defined under tensile loading. Firstly, material behaviour was characterized using classic DMTA. Secondly, several DSAs were applied in order to observe and discuss the variations in material behaviour with respect to the number of cycles. Once the mechanical behaviour had been characterized at various DSAs, it became possible to discuss the evolution of the Payne effect at a given number of cycles. The effect 
of temperature was also investigated by performing the experiments at ambient temperature and $80^{\circ} \mathrm{C}$. A state of the art appraisal of the effect of fillers on the viscoelastic properties of rubber is presented initially. The material, the specimen geometry and the loading conditions are then detailed, and the results obtained are discussed. Finally, some perspectives are given for future work.

\section{State of the art appraisal}

Many theories have been proposed in the literature to discuss the viscoelastic response of rubber, but the physical mechanisms involved are still not clearly established. Nevertheless, several phenomena induced by the matrix and the fillers, as well as their interactions, are classically considered:

1. the formation of a filler network comprising filler-filler and filler-matrix interactions (Wang, 1999);

2. the existence of occluded polymer located within and between the filler aggregates, which increases the effective filler concentration (Morozov et al., 2010);

3. the formation of a layer of bound polymer around the filler particle surface (Morozov et al., 2010);

4. the interactions between fillers and matrix along the interface, which leads to adsorption and desorption of polymer chains at several mobility levels (Maier and Goritz, 1996);

5. the hydrodynamic effect (Einstein, 1906).

The first phenomenon quoted is activated when the filler fraction is sufficient to form a network, composed of agglomerates, i.e. clusters of aggregates, which are bound to each other by physical linkages (Wang, 1999; Medalia, 1978). During cyclic loading, this network successively disrupts and reforms. Thus, the evolution of the competition between the disruptions and reformations of this filler network drives the variations in the viscoelastic properties, more precisely the decrease in the storage modulus with an increasing DSA, which is called the Payne effect (Payne, 1962; Wang, 1999; Voet, 1975). Wang claims that this competition is the main, or even the sole cause of the Payne effect (Wang, 1999). Concerning the network of carbon black filler itself, Morozov et al. (2010) have shown that the filler network exists in four states, which depend on filler concentration:

- separates aggregates;

- individual clusters;

- cluster network with dense regions of cluster cores and aggregate branches linking neighbouring secondary structures;

- continuous filler structure.

It should be noted that the strength of the filler network also depends on the type of filler (Wang, 1999), more precisely on the filler surface characteristics (Wang et al., 1991a,b).

Some morphological features, like bound and occluded polymer volume fractions, depend on the state of the filler network. As mentioned above, disruption and reformation occur during mechanical cycles. If the filler network does not have enough time to recover its original structure during one cycle, $E^{\prime}$ begins to decrease. This phenomenon occurs once the DSA is sufficient to disturb the balance between disruption and reformation, and therefore ends once the filler network is completely disrupted. Consequently, the evolution of the filler network structure can be partly responsible for the variations in material behaviour for the intermediate strain amplitude values. The breakdown of the filler network allows the release of occluded rubber, which was originally trapped within the filler aggregates and contributed as a filler to the mechanical behaviour of the rubber. Occluded rubber may be a factor in the high initial level of $E^{\prime}$ and its release plays a role in the decrease in $E^{\prime}$. Another reason for the difference in $E^{\prime}$ level between filled and unfilled rubbers resides in the polymer layer that surrounds the filler particles (Litvinov and Steeman, 1999). This layer contains:

- polymer chains in the glassy state;

- polymer chains with more mobility near the external borders.

This locally causes the density of the matrix cross-linkage to increase. Thus, the disentanglement of the polymer chains, which is initiated by straining, contributes to the increase in $E^{\prime}$ for small DSA ranges. Despite the numerous studies reported in the literature dealing with the Payne effect, the influence of the evolution of the matrix itself, as well as that of the fillers, on the viscoelastic properties of rubber when it is subjected to a high number of cycles $\left(10^{6}\right.$ cycles) has never been investigated till now. This type of variation in the rubber microstructure is more commonly studied by chemists, who, using DMA or DMTA, tend to characterize the evolution of the microstructure when the material is subjected to photo-oxidation (Feller, 1994) or thermo-oxidation (Kumar et al., 2004) for instance. Even though these studies have improved knowledge of the mechanisms involved, none of them investigates the effect of the mechanical cycles on the microstructure itself and therefore on the evolution of the viscoelastic properties of rubber. This is the aim of the research described here.

\section{Experimental setup}

\subsection{Materials and specimen geometry}

The materials tested in this study are nitrile rubbers, respectively unfilled and filled with $35 \mathrm{phr}^{1}$ of carbon black and of a small amount of mineral fillers. They were vulcanized with sulphur. The following procedure was used to prepare the specimens: (i) a block was first obtained by compression moulding; (ii) which was then cut with a water jet cutting device to obtain specimens of $5 \times 5 \mathrm{~mm}^{2}$ square cross-section and $10 \mathrm{~mm}$ height; (iii) the resulting low aspect ratio was chosen in such a way that buckling, which might have occurred during the compressive tests, was

\footnotetext{
1 Parts per hundred parts of rubber in weight.
} 
avoided. Finally, it is worth noting that this type of cutting technology (water jet cutting) avoids microstructural damage on the surface of the specimen.

\subsection{Dynamic mechanical and thermal analysis}

The dynamic properties of rubber were measured by means of a Metravib VA2000 viscoanalyzer. First, the specimen to be tested was glued with cyano-acrylate glue onto plates fixed to grips. Uniaxial cyclic loading was then applied to the specimen via a shaker through the upper grip. A force sensor located under the lower grip recorded the force signal transmitted through the specimen, while a displacement sensor measured the displacement of the upper grip. The tests were performed under controlled displacement.

\subsubsection{Loading conditions}

Classic DMTA was first carried out with filled and unfilled nitrile rubbers. The procedure consisted in first applying an increasing DSA sweep from 0.1 to $15 \%$, and then a decreasing DSA sweep from 15 to $0.1 \%$. DSA sampling satisfied a logarithmic distribution, as in Refs. (Wang, 1999; Rendek and Lion, 2010). This choice is justified by the fact that the Payne effect is the highest for the lowest DSA value. The cycle defined by an increasing DSA sweep, followed by a decreasing DSA sweep, was repeated ten times in order to examine the stabilized viscoelastic response. The tests were performed at a constant frequency of $10 \mathrm{~Hz}$. The influence of temperature on the mechanical response was assessed by performing tests at ambient temperature $\left(20^{\circ} \mathrm{C}\right.$ in the present case) and $80^{\circ} \mathrm{C}$. Tensile and compressive loading conditions were applied. In both cases, the mechanical properties were evaluated in terms of $E^{\prime}, E^{\prime \prime}$ and $\tan (\delta)$. Their variations were also recorded during the tests.

For the cyclic loading tests at a constant DSA, which were performed here under compressive loading conditions, the displacement applied to the specimens was sinusoidal, with a constant frequency of $10 \mathrm{~Hz}$. The maximum value of this displacement was negative, i.e. the loading case corresponded to uniaxial compression/compression loading conditions. Two series of tests were performed, each at a constant temperature: $20^{\circ} \mathrm{C}$ and $80^{\circ} \mathrm{C}$. Each test was performed up to one million cycles, at a constant DSA. The duration of each test was about $28 \mathrm{~h}$. The displacement applied was such that it corresponded again to a range of $0.1 \%$ to $15 \%$ DSA during all the tests, for consistency with the previous tests. Ten tests were carried out for both series, and sampling was the same as previously. In practice, this corresponded to $0.1,0.17,0.3,0.92,1.61,2.8,4.9$, 8.6 and $15 \%$ DSA.

\section{Results}

\subsection{Classic DMTA}

\subsubsection{Tensile loading conditions}

Filled nitrile rubber. As explained previously, the tests consisted of applying two DSA sweeps, the first from 0.1 to $15 \%$, the second from 15 to $0.1 \%$, for filled nitrile rubber at both ambient temperature and $80^{\circ} \mathrm{C}$. Fig. 1(a) shows the variation in $E^{\prime} v s$. DSA observed during the first ten cycles. For the sake of clarity, only four representative cycles are shown, cycles $1,5,7$ and 10 . At ambient temperature, $E^{\prime}$ decreases when the DSA increases for both the loading and unloading sweeps. This is the classic representation of the so-called "Payne effect". The unloading curves are always located under the loading curves. Contrary to cycle 1 , the unloading curve meets the loading curve at low DSA for cycles 2 to 10 , thus giving rise to hysteresis loops. The shape of these loops is stabilized from the seventh cycle onward. The highest level of $E^{\prime}$ is obtained for the loading sweep of cycle 1. Moreover, the highest level of irreversibility is obtained during the first cycle, with a significant set in terms of $E^{\prime}$ loss. A plateau is observed for small DSA values (<0.16\% DSA) for each loading curve. This phenomenon is not observed for the unloading curve. In addition, the gap between the successive loading curves and successive unloading curves is more significant for small DSAs. Every loading and unloading curves reaches the same value at the highest DSA.

At $80^{\circ} \mathrm{C}$, the loading and unloading curves only differ for cycle 1 . No irreversibility is observed for the subsequent cycles. The response is stabilized from cycle 2 onward, and the applied DSA has no significant effect on $E^{\prime}$. Hence, the Payne effect is not observed at this temperature level and for this filler volume fraction. Nevertheless, some experiments have shown that the Payne effect remains observable at this temperature if the filler concentration is greater (see for example the results given in Ref. Wang, 1999). According to the mechanisms discussed previously, the injected heat increases local macromolecular mobility and consequently acts in similarly to an increase in DSA.

Fig. 1(b) shows the corresponding variation in $\tan \delta v s$. DSA. At ambient temperature, similar to $E^{\prime}$, the loading and unloading curves differ and form a hysteresis loop. A plateau is obtained for a DSA equal to 0.1 to $2.8 \%$ where $\tan \delta$ lies between 0.4 and 0.5 . After a slight increase for the loading sweeps, a decrease is observed from this maximum value to 0.3 . This global maximum is obtained at a DSA for which the viscous dissipation is maximum. Indeed, most of the occluded rubber is released and cyclic disruptions and reformations of the filler network also contribute to the energy dissipation. At $80^{\circ} \mathrm{C}$, the level of $\tan \delta$ is about 3 times lower than that observed at ambient temperature. $\tan \delta$ remains constant, so no hysteresis loop is observed.

Unfilled nitrile rubber. Classically, the Payne effect is considered as a consequence of the addition of reinforcing fillers to the compound. Consequently, unfilled rubber is not expected to exhibit such a response. Results obtained with unfilled nitrile rubber are reported in this section. They are briefly presented and discussed in the light of analyses available in the literature. The tests performed were the same as those carried out with filled nitrile rubber. Fig. 2(a) gives the variation in $E^{\prime} v s$. DSA obtained during the cycles at both the ambient temperature and $80^{\circ} \mathrm{C}$. At ambient temperature, results are similar to those obtained for filled nitrile rubber at $80^{\circ} \mathrm{C}$. Indeed, an increase in temperature increases the macromolecular mobility of the polymer chains in the vicinity of the filler aggregates (Berriot et al., 2002, 2003; Merabia et al., 2008). This 

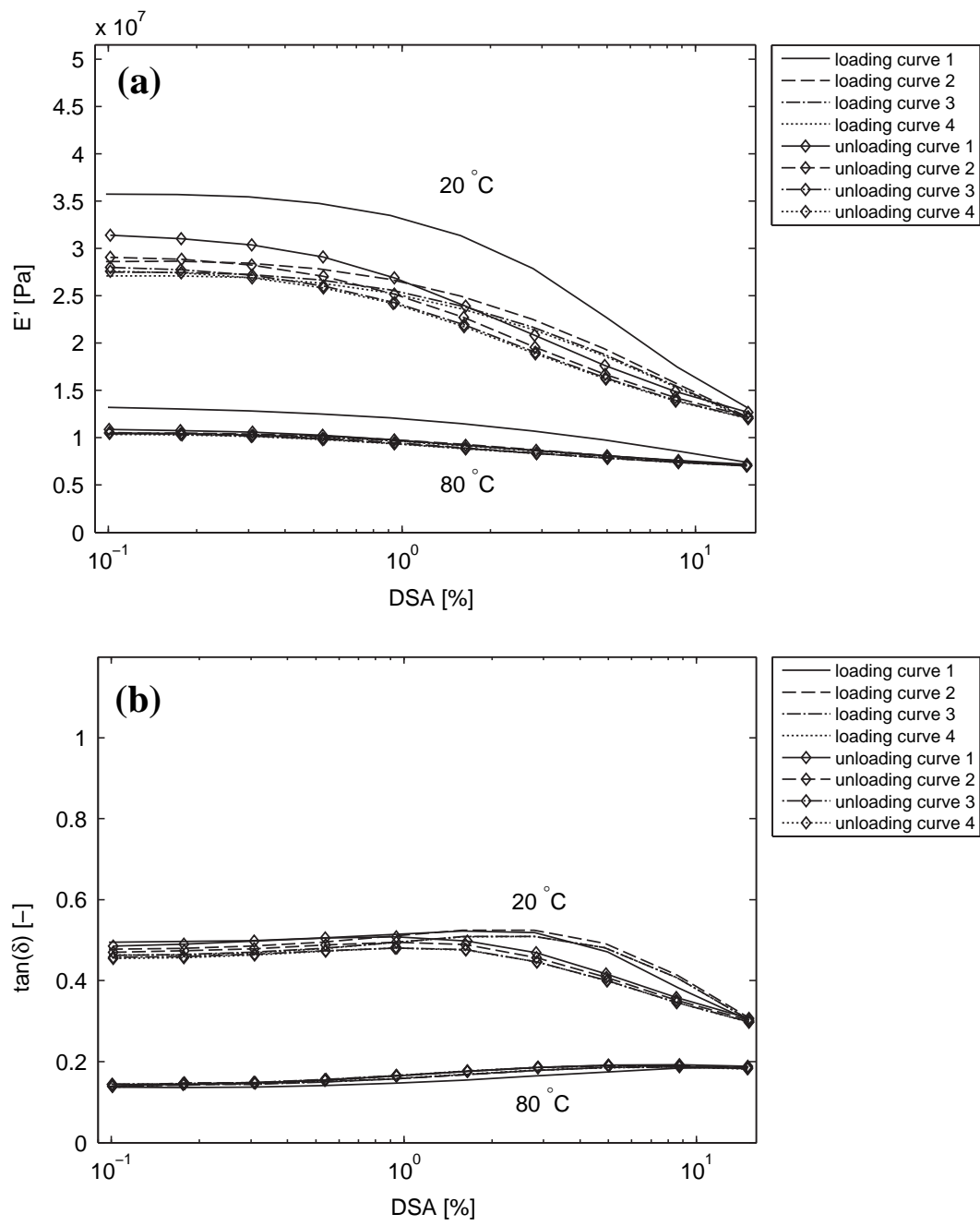

Fig. 1. $E^{\prime}(\mathrm{a})$ and $\tan \delta$ (b) of filled nitrile rubber $v$ s. DSA during cyclic sweeps at both ambient temperature and $80^{\circ} \mathrm{C}$ under tensile loading conditions.

weakens the effect of fillers. Consequently, the Payne effect tends to vanish. Moreover, the fact that the $E^{\prime}$ levels obtained in both cases are close to each other probably indicates that the hydrodynamic effect is impaired by a temperature increase. At $80^{\circ} \mathrm{C}, E^{\prime}$ does not depend on the DSA and is not affected by the number of cycles.

The evolution of $\tan \delta$ can be related to the variation in the ratio of the part of the filler network that is able to disrupt and reform and the remaining part which is stable during straining. Thus, the maximum value occurs at a DSA where the dissipative phenomena are the greatest. According to the variations in $\tan \delta$, the shape of the hysteresis loop is the same as for filled rubber, but a shift of the curves is observed. This corresponds to a decrease in $\tan \delta$. At $80^{\circ} \mathrm{C}$, this quantity does not depend on the DSA. It evolves in a manner similar (in shape and magnitude) to that of filled rubber at the same temperature.

\subsubsection{Compressive loading conditions}

Filled nitrile rubber. The four tests described in Section 4.1.1 were repeated, but using compressive loading condi- tions to observe a possible influence of this type of load. The first difference compared to the tensile strain sweeps lies in the values attained for each test. Indeed, Fig. 3(a) gives the variation in $E^{\prime}$ at both the ambient temperature and $80^{\circ} \mathrm{C}$. The fact that $E^{\prime}$ is higher in this case than under tensile loading conditions may be explained by the fact that the strain field is less homogeneous in the compressive case. Moreover, the phenomena observed for the tensile loading conditions discussed above show that for each DSA of loading and unloading sweep, the level of $E^{\prime}$ continuously decreases, cycle after cycle. This monotony is not observed during the compressive strain sweeps. Indeed, the evolution of $E^{\prime}$ during loading and unloading sweeps exhibits a maximum (during the fourth or fifth cycle), and then decreases to reach a value which is below the level of the curves corresponding to the first cycle.

Concerning the variation in $\tan \delta$ at ambient temperature, Fig. 3(b) shows that, contrary to the case of tensile loading conditions, there is no hysteresis loop in the current case of compressive loading conditions. For both the loading and unloading sweeps, non-monotony in the 

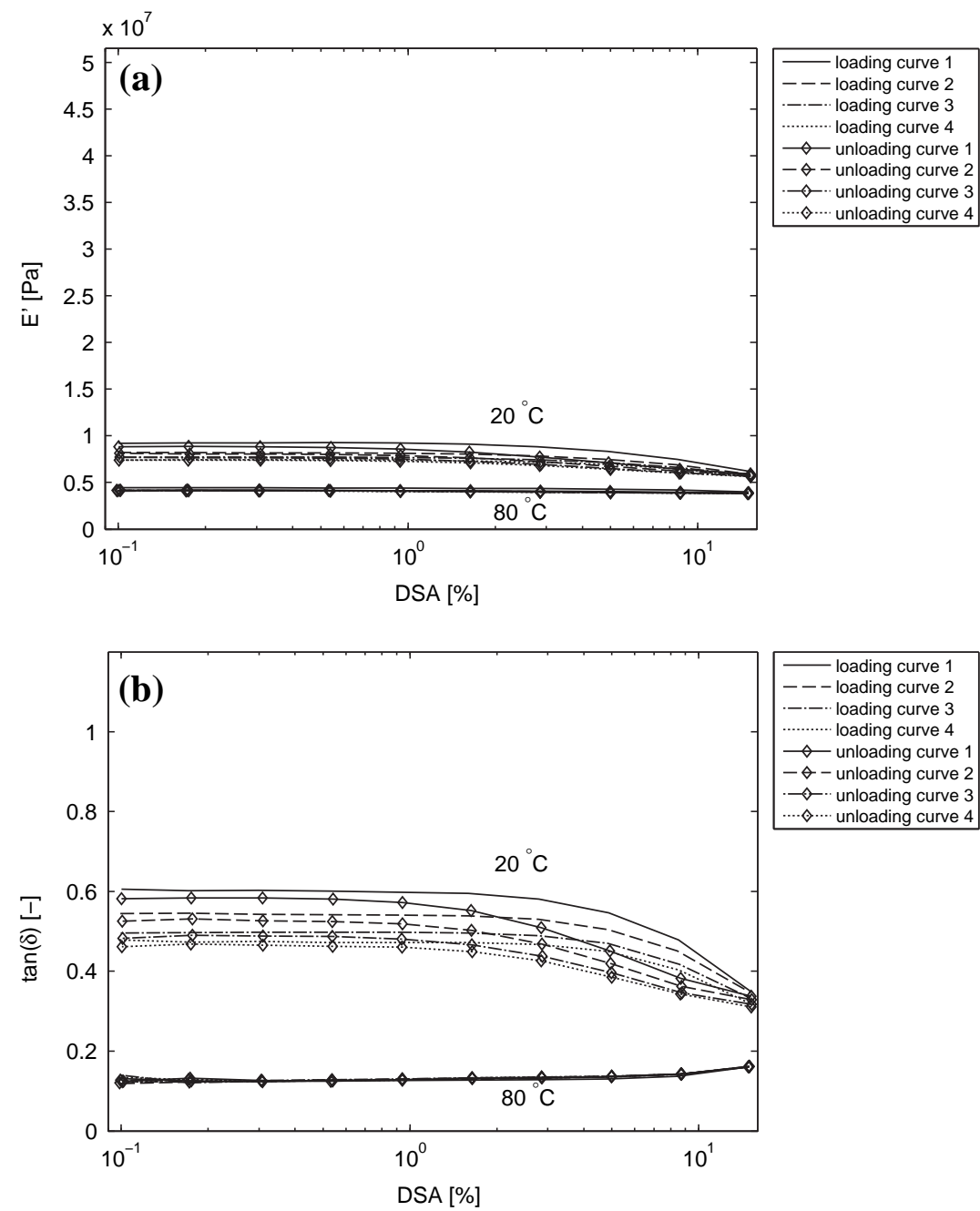

Fig. 2. $E^{\prime}$ (a) and $\tan \delta$ (b) of unfilled nitrile rubber vs. DSA during cyclic sweeps at both ambient temperature and $80^{\circ} \mathrm{C}$ under tensile loading conditions.

relative curve position is observed. Finally, at $80^{\circ} \mathrm{C}$, the results are the same as those obtained under tensile loading conditions.

Unfilled nitrile rubber. Fig. 4(a) and (b) give the variation in $E^{\prime}$ and $\tan \delta$ of unfilled rubber at both temperatures. As for the filled nitrile rubber, $E^{\prime}$ at ambient temperature is globally higher when using compressive loading conditions than when using tensile loading conditions. Moreover, non-monotony is observed for both the loading and unloading curves. However, the general trend is the same as that observed for the tensile tests. Again, $E^{\prime}$ is independent of DSA. At ambient temperature, the general trend for $\tan \delta$ is very similar to that observed for the tensile loading conditions at the same temperature. The differences lie in the fact that the hysteresis loop is lower between the loading and unloading curves and that there is no observed maximum value after the plateau for the loading curves. At $80^{\circ} \mathrm{C}, \tan \delta$ is independent of DSA.

As a summary, in the light of the DMTA experiments carried out on nitrile rubber, the Payne effect is characterized with respect to both the temperature variation and the presence or not of carbon black fillers ( $35 \mathrm{phr}$ ). The results obtained are in good agreement with those reported in the literature (Wang, 1999; Rendek and Lion, 2010; Medalia, 1978). The temperature increase leads to a thermal activation which allows the macromolecules to slip easily between each other and within the agglomerates. The initial stiffness also reduces. Finally, the type of loading conditions changes both the relative position between successive sweeps and the values of $E^{\prime}$, but the Payne effect is virtually unchanged.

\subsection{Cyclic loading tests at ambient temperature}

The effect of mechanical cycles on viscoelastic properties is addressed in this section. Since the previous section showed that fillers influence the viscoelastic properties of the material, only the filled nitrile rubber is examined here.

Fig. 5 shows the variation in $E^{\prime}$ at ambient temperature during the tests. Each DSA applied previously was applied again, but for the sake of clarity, only four typical curves are plotted in the diagram. The first significant finding is 

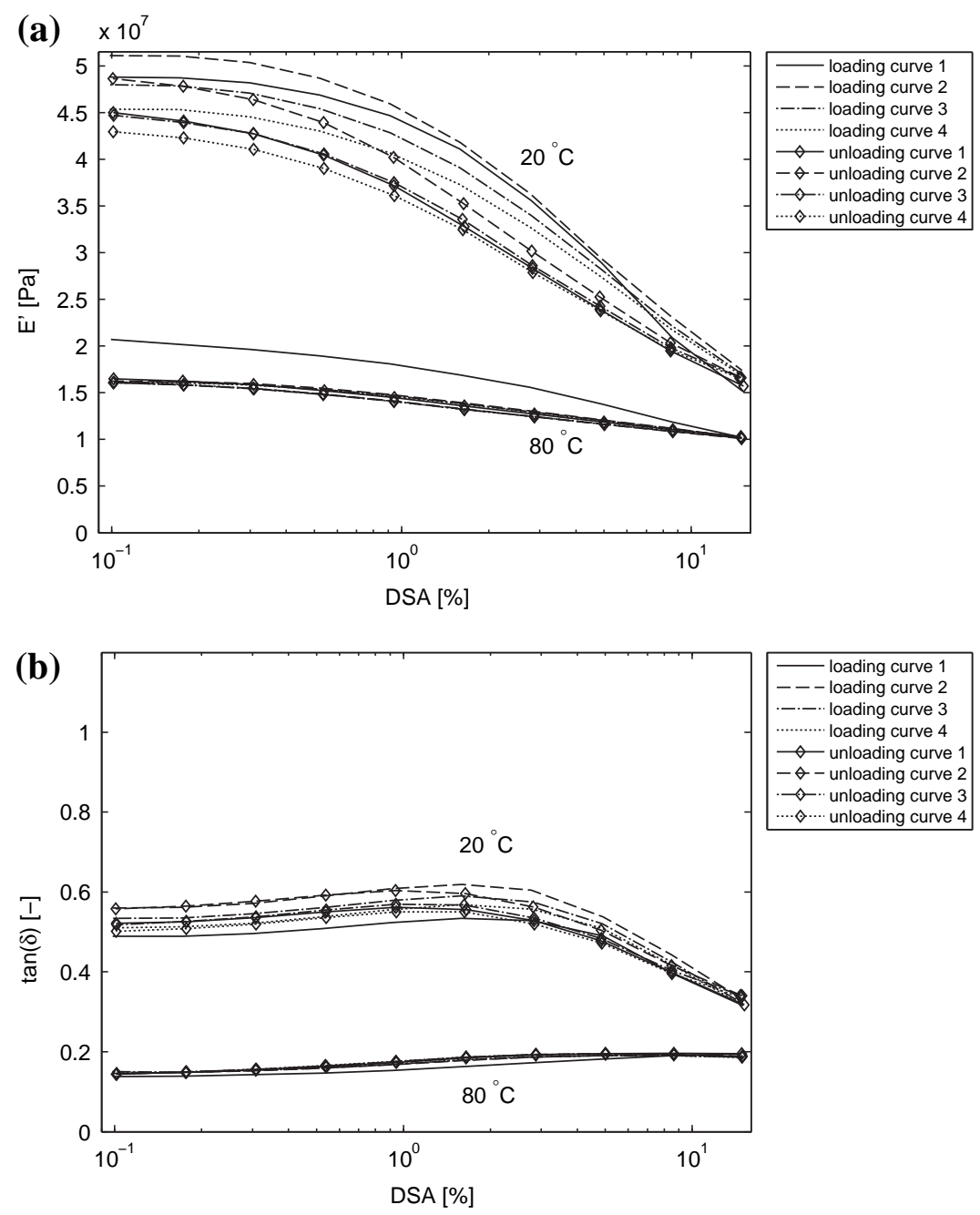

Fig. 3. $E^{\prime}(\mathrm{a})$ and $\tan \delta$ (b) of filled nitrile rubber $v s$. DSA during cyclic sweeps at both ambient temperature and $80{ }^{\circ} \mathrm{C}$ under compressive loading conditions.

that whatever the number of cycles, the higher the DSA, the lower the storage modulus. This is a first analogy with the Payne effect discussed previously. The second observation is that apart from the highest DSA level (15\%), the initial level of $E^{\prime}$ is lower than the final one. This is somewhat unusual, since cyclic mechanical loading generally induces a decrease in $E^{\prime}$. Another interesting feature is that all the curves obtained can be seen as a succession of five typical elementary steps, which are shown in Fig. 6 for the particular case of $2.8 \%$ DSA.

The first step, called step 1 in the following, only occurs during the very early stages of the tests. It corresponds to a decrease in $E^{\prime}$, which is observed in Fig. 7. This Figure is a magnification of the boxed zone shown in Fig. 6 for the first few thousand cycles. This decrease in $E^{\prime}$ is observed up to $1240,450,1000$ and 3000 cycles for $1.61,2.8,4.9$ and $15 \%$ DSA, respectively. These threshold values do not depend on the applied DSA. This first step can be considered as a material softening. The combined variations in both $E^{\prime}$ (see Fig. 5) and $\tan \delta$ (see Fig. 8) during the four following steps are more clearly visible by normalizing the curves.
This normalization is performed by dividing $E^{\prime}$ and $\tan \delta$ by their respective maximum values. The corresponding diagram, illustrating the normalization for the particular case of $2.8 \%$ DSA, is given in Fig. 9. After a decrease in $E^{\prime}$ during step 1, step 2 corresponds to a strong increase up to a maximum value reached at $4.5 \times 10^{4}, 9.7 \times 10^{4}$, $5.6 \times 10^{4}, 2.3 \times 10^{4}$ cycles for $1.61,2.8,4.9$ and $15 \%$ DSA, respectively. It is worth noting that the maximum value of $E^{\prime}$ reached at the end of step 2 is higher than that observed at the beginning of the test, thus signifying that the material becomes stiffer than in its initial state. Step 3 corresponds to a decrease in $E^{\prime}$. It is longer than step 2 . Step 4 corresponds to an increase in $E^{\prime}$. This is longer than step 3. Fig. 6 shows that the material hardening rate during step 4 is lower than both the softening rate in step 3 and the hardening rate in step 2. Finally, step 5 corresponds to a decrease in $E^{\prime}$. The limits of the steps, in terms of the number of cycles, depend on the DSA. These results clearly illustrate the complexity of the mechanical response of the filled nitrile rubber under cyclic loading. This emphasizes the fact that the material is simultaneously subjected to 

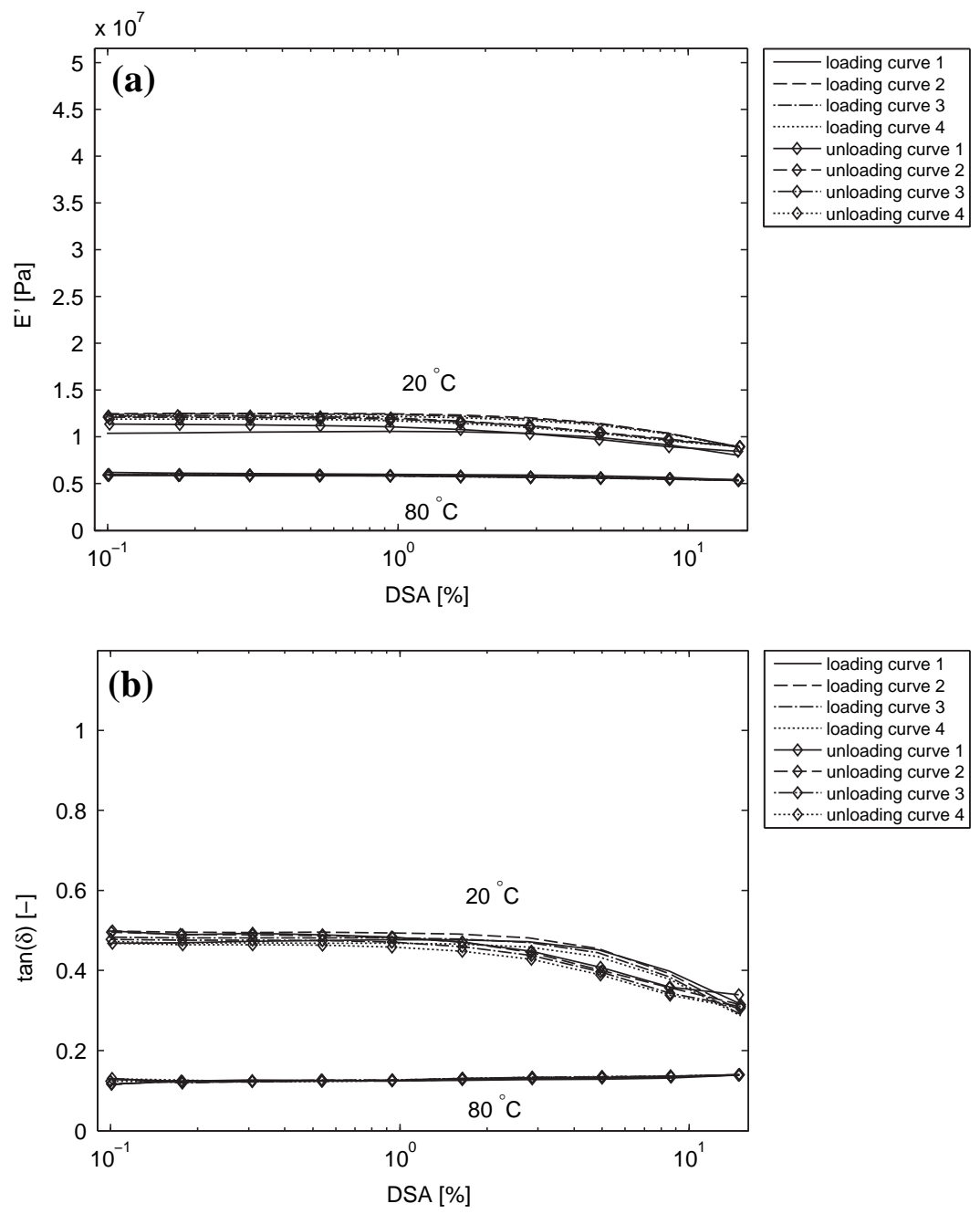

Fig. 4. $E^{\prime}$ (a) and $\tan \delta$ (b) of unfilled nitrile rubber vs. DSA during cyclic sweeps at both ambient temperature and $80{ }^{\circ} \mathrm{C}$ under compressive loading conditions.

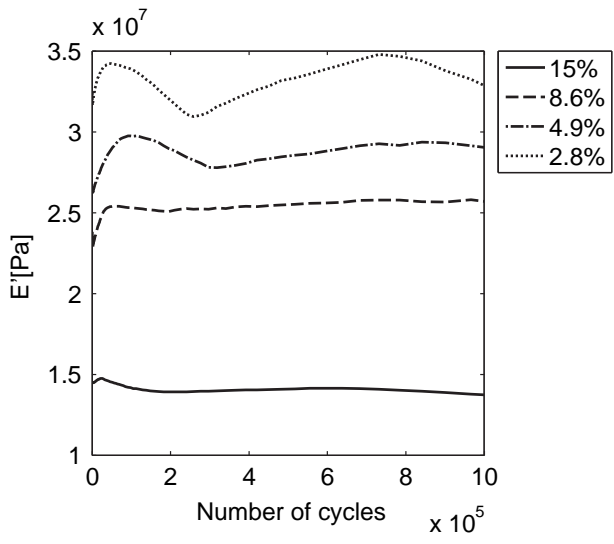

Fig. 5. $E^{\prime}$ versus the number of cycles at ambient temperature, for the four DSA considered.

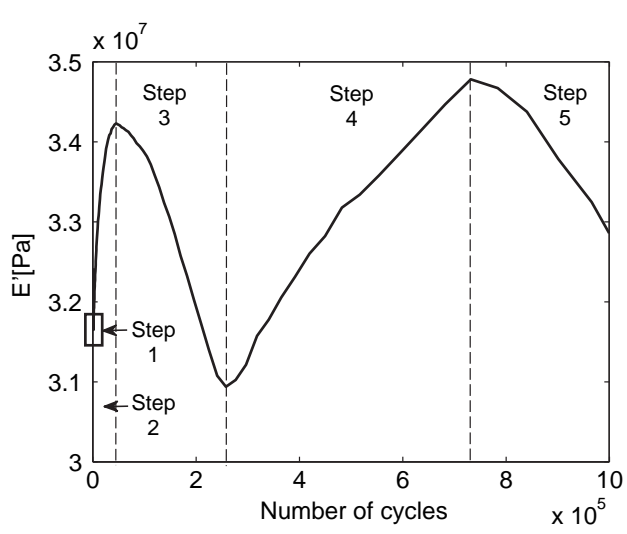

Fig. 6. Elementary steps of a fatigue test illustrated at $2.8 \%$ DSA at ambient temperature. 

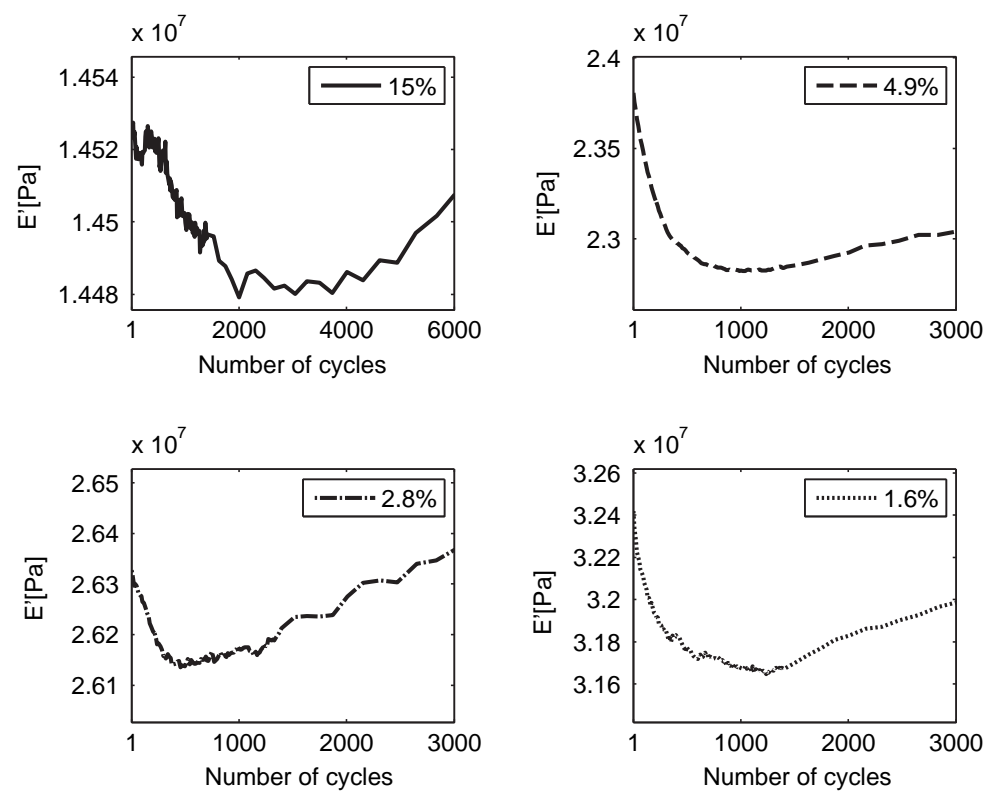

Fig. 7. Magnified view of $E^{\prime}$ on the first thousands of cycles carried out at ambient temperature for the four DSA considered.

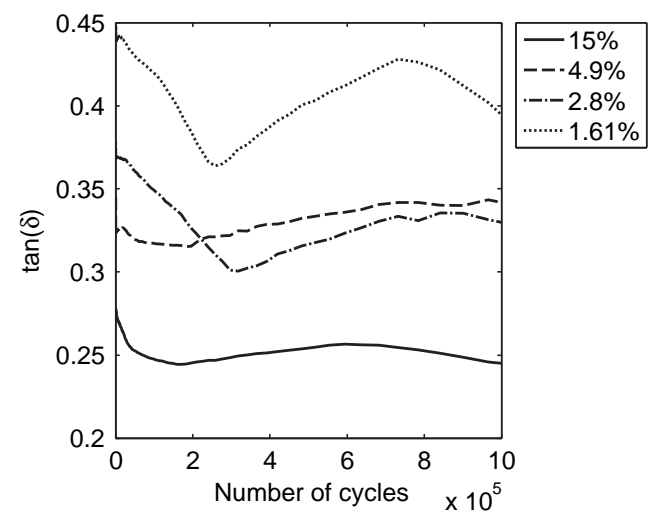

Fig. 8. $\tan \delta$ vs. the number of cycles for the four DSA considered at ambient temperature;

both physical and chemical variations. With classic DMTA, the viscoelastic response of rubber depends on the cyclic disruption and reformation of the filler network, as well as on the possible release of occluded rubber (Wang, 1999). Under a higher number of cycles, some additional phenomena occur, such as fatigue of the filler network and likely chemical variations in the rubber matrix (e.g. vulcanization, chain scissions Feller, 1994).

Figs. 8 and 9 are presented in order to discuss the mechanisms involved during the five steps. For steps 1,3 , 4 and 5 , the variation in the loss factor is similar to that of $E^{\prime}$, i.e. viscosity increases as $E^{\prime}$ increases, and then decreases as $E^{\prime}$ decreases.

Step 2 is now considered again for comparison purposes with step 4 . Similarly to step 4 , step 2 corresponds to material hardening, but while the loss factor increases during step 4 , either it begins to increase before decreasing during

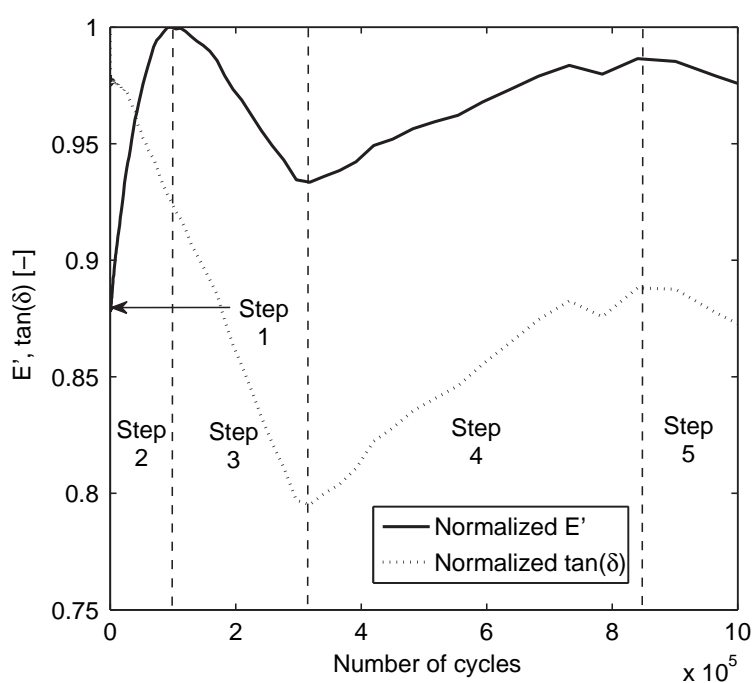

Fig. 9. Superimposition of normalized $E^{\prime}$ and $\tan \delta$ for $2.8 \%$ DSA at ambient temperature.

step 2 (for 1.61 and $4.9 \%$ DSA) or it first slowly and then more quickly (for 2.8 and $15 \%$ DSA) decreases as shown in Fig. 8. This is clearly visible in Fig. 9 where both the normalized values of $E^{\prime}$ and $\tan \delta$ are represented: during steps 2 and $3, \tan \delta$ regularly decreases while $E^{\prime}$ increases during step 2 and then decreases during step 3. Conversely, during step 4, both $E^{\prime}$ and $\tan \delta$ increase. This illustrates that the hardening mechanism is different for steps 2 and 4 . As mentioned above, $E^{\prime}$ significantly increases during step 2 . This can be explained by several scenarios. The first is that this increase in $E^{\prime}$ can be attributed to the vulcanization of the rubber matrix. However, $\tan \delta$ still decreases in step 3 and $E^{\prime}$ should continue to increase instead of decreasing. 
Consequently, it is believed that this scenario is not the most realistic. Another explanation is given by considering the influence of the reinforcing fillers on the mechanical response of the material. To shed light on the cause of this phenomenon, one of the experiments described above was performed on an unfilled rubber specimen. A higher DSA was used (15\% DSA), to try to reflect the fact that macromolecules attain a local stress level. This stress level is certainly significantly higher than that reached by the macromolecules in the unfilled material, because fillers act as local stress concentrators in the filled material (Suzuki et al., 2005). Fig. 10 compares the responses of a filled and an unfilled rubber specimen. It is clear that the response of the unfilled rubber specimen is not the same as that of the filled one, since $E^{\prime}$ continuously decreases up to $1.7 \times 10^{5}$ cycles for the unfilled material only. Therefore it is possible to conclude that the fillers are the cause of the phenomenon discussed here. To the best of the authors' knowledge, this phenomenon concerning cyclic loading with low DSA has not been reported in the literature until now.

Finally, similar results can be observed concerning the variation in the normalized value of $E^{\prime}$ at a higher number of cycles. The fact that the normalized value of $E^{\prime}$ increases

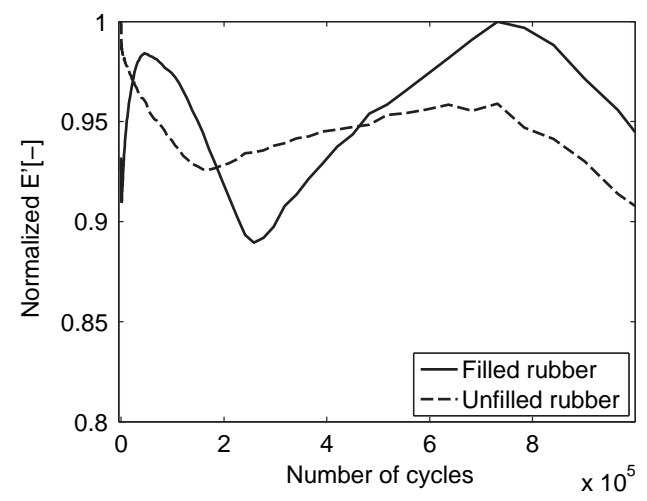

Fig. 10. Superimposition of normalized $E^{\prime}$ of filled and unfilled nitrile rubber at 1.6 and $15 \%$ DSA respectively at ambient temperature.

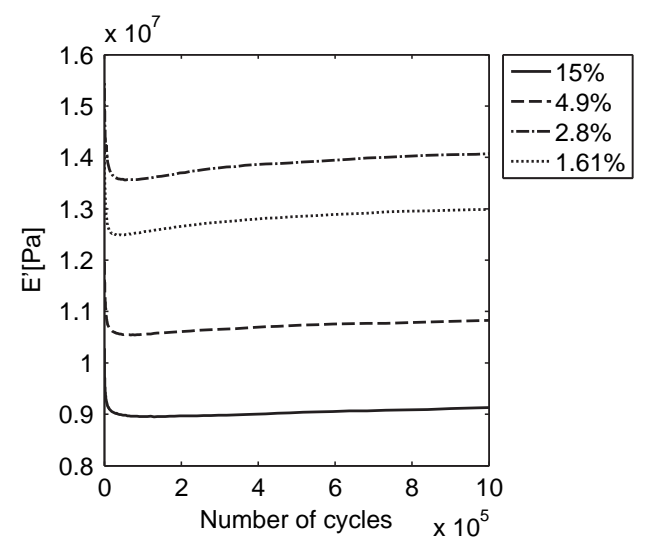

Fig. 11. $E^{\prime}$ vs. the number of cycles at $80^{\circ} \mathrm{C}$. and then decreases may be due to the macromolecular network. It is clear that it can be due neither to the filler network nor to its interaction with the macromolecular network, since this phenomenon can be observed in both the filled and unfilled rubbers.

It must be emphasized that a stabilized response is generally considered to be indicative of the behaviour of the material at high number of cycles (Brunac et al., 2009; Andriyana et al., 2010). The current study clearly shows that this assumption might not be accurate enough, because it does not take into account the microstructural evolution of rubber, at least for the material studied in the present work.

\subsection{Cyclic loading tests at $80^{\circ} \mathrm{C}$}

The effect of temperature on the viscoelastic response of filled nitrile rubber under cyclic loading is investigated in this section. The tests described previously were performed at $80^{\circ} \mathrm{C}$ for this purpose.

Fig. 11 gives the variation in $E^{\prime}$ during the tests. The curves observed in this case are simpler to those obtained at ambient temperature. Firstly, each curve exhibits the same general shape. Secondly, the viscoelastic response can be split into two steps. The first step corresponds to a strong decrease in $E^{\prime}$ during the first $5 \times 10^{4}$ cycles. This

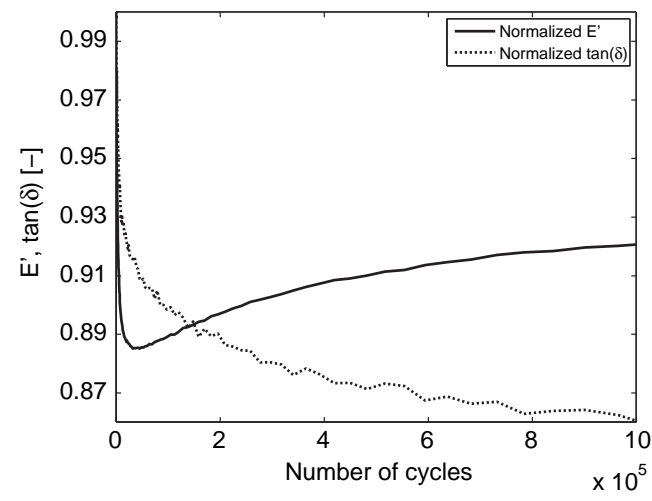

Fig. 12. Normalized $\tan \delta$ and $E^{\prime}$ for $4.9 \%$ DSA at $80^{\circ} \mathrm{C}$.

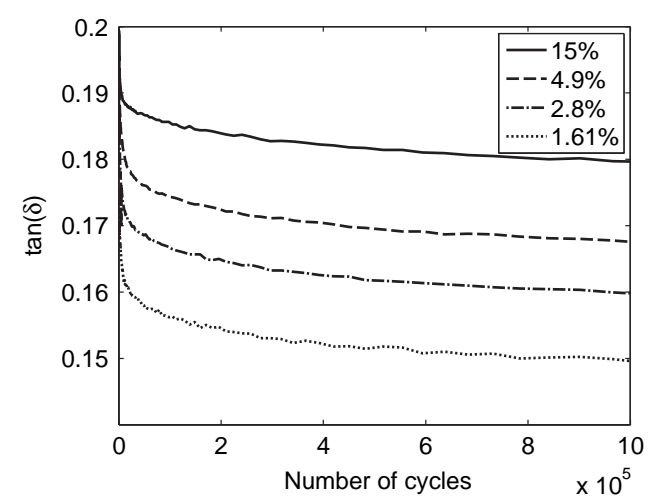

Fig. 13. $\tan \delta v s$. the number of cycles at $80^{\circ} \mathrm{C}$. 

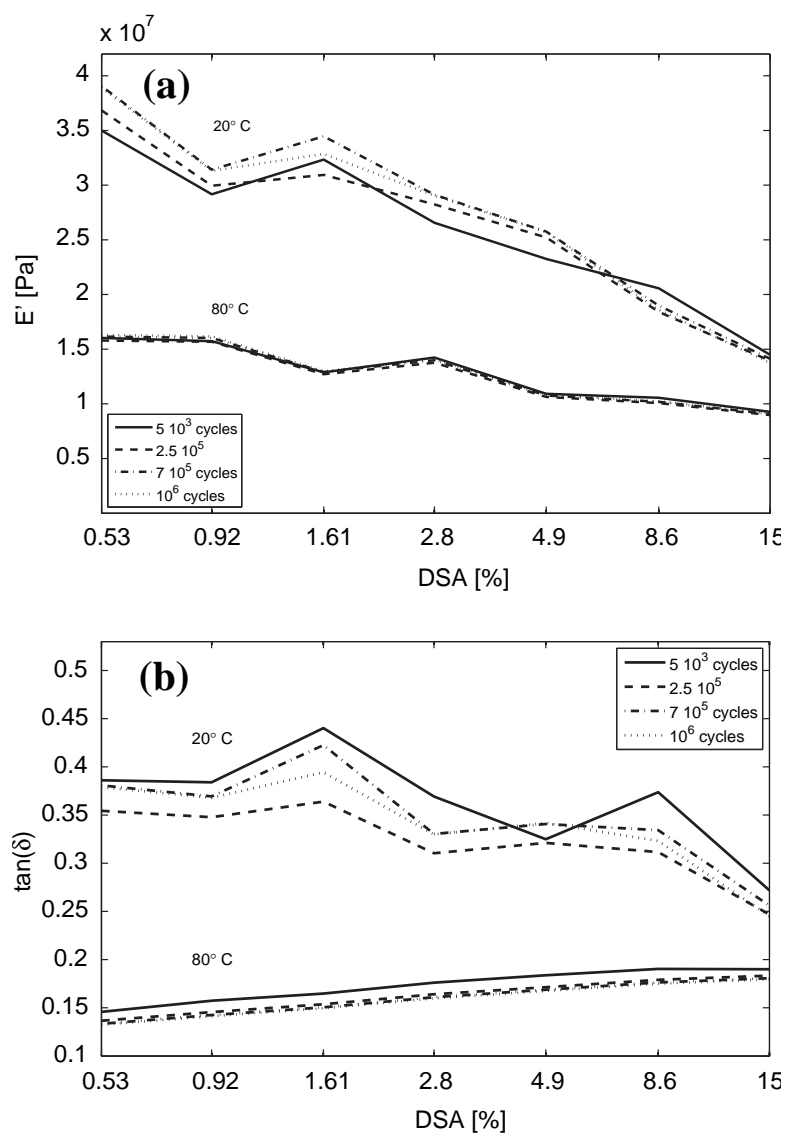

Fig. 14. $E^{\prime}$ (a) and $\tan \delta$ (b) of filled nitrile rubber vs. DSA for different number of cycles at both ambient temperature and $80^{\circ} \mathrm{C}$ using compression.

is due to the combination of viscosity and material softening, which is a well-known phenomenon. Then, during the second step, $E^{\prime}$ slightly and continuously increases. Fig. 12 shows a superimposition of the normalized value of $E^{\prime}$ and $\tan \delta v s$. the number of cycles for $4.9 \%$ DSA. $\tan \delta$ (Fig. 13) continuously decreases, firstly significantly during the initial step and then less markedly during the second step. The fact that $E^{\prime}$ increases and $\tan \delta$ decreases during the second step tends to show that the material has been subjected to vulcanization. As a high temperature tends to reduce the effect of fillers, the fact that an increase in $E^{\prime}$ is not observed during the first thousand cycles confirms that step 2 in Fig. 9 depicts a situation that is due to fillers.

\section{Influence of the number of cycles on the Payne effect}

In the previous sections, the evolution of certain mechanical quantities, viz. $E^{\prime}$ and $\tan \delta$, was shown vs. the number of cycles. The Payne effect is classically represented in the $E^{\prime}$-DSA diagram. Various curves showing the variation in $E^{\prime}$ and $\tan \delta v s$. DSA are therefore plotted at different stages of the tests to characterize and discuss the influence of the number of cycles on the Payne effect at both ambient temperature and $80^{\circ} \mathrm{C}$.
Fig. 14 shows some $E^{\prime}$-DSA curves obtained for various numbers of cycles. Four typical values for the number of cycles were chosen: $5 \times 10^{2}, 2.5 \times 10^{5}, 7 \times 10^{5}$ and $10^{6} \mathrm{cy}-$ cles. The following comments can be made.

Firstly, for both temperatures, the number of cycles does not modify the general shape of the curve. This may indicate that the Payne effect is not altered by the number of cycles or the temperature.

Due to the fact that the step duration is different for each DSA level, it is difficult to compare quantitatively the relative positions of the curves. Nevertheless, it clearly appears that at ambient temperature, the gap between the curves observed for low DSA is larger than that for higher DSA. These curves tend to the same value at $15 \%$ DSA. This means that the higher the DSA level, the smaller the influence of the number of cycles. Indeed, at high DSA the filler network no longer has any influence on the mechanical response. At $80^{\circ} \mathrm{C}$, this phenomenon is no longer observed and the curves are superimposed. As observed during classic DMTA, DSA has only a small effect on $E^{\prime}$. This is certainly the reason why no difference is observed between the curves for different numbers of cycles.

\section{Conclusion}

The effect of cyclic loading on the viscoelastic properties of filled and unfilled nitrile rubbers is addressed in this study.

Classic DMTA has confirmed that the viscoelastic response of the filled nitrile rubber under study, submitted to tensile and compressive loading conditions, exhibits features previously reported in the literature.

The following conclusions can be drawn from the cyclic tests performed for several DSA at both ambient temperature and $80^{\circ} \mathrm{C}$ :

1. the viscoelastic properties of the filled rubber clearly evolve during the cyclic loading tests;

2. in filled nitrile rubber, at ambient temperature and after the stress softening step, $E^{\prime}$ is greater than its initial value, apart from at the highest DSA. This phenomenon is not observed in unfilled nitrile rubber. This tends to prove that it is caused by the filler network;

3. at $80^{\circ} \mathrm{C}$ and for each DSA, $E^{\prime}$ slowly increases after a short softening phase. This is observed until the end of each test. This increase in stiffness seems to reveal a progressive vulcanization of the filled rubber;

4. The Payne effect is not really modified, even from a quantitative point of view, by the number of cycles.

The first two points should be considered to allow improved prediction of variations in viscoelastic behaviour during cyclic loading. Further work in this field is currently being carried out by the authors.

\section{Acknowledgements}

The PCM Company is acknowledged for its financial support. The authors are also very grateful to Dr. Berton, Mrs. Serres, M Perret, M Chatelain and M Fay for fruitful discussions. 


\section{References}

Andriyana, A., Saintier, N., Verron, E., 2010. Configurational mechanics and critical plane approach: concept and application to fatigue failure analysis of rubberlike materials. Int. J. Fatigue 32 (10), 1627-1638.

Bergström, J.S., Boyce, M.C., 2000. Large strain time-dependent behavior of filled elastomers. Mech. Mater. 32, 627-644.

Berriot, J., Montes, H., Lequeux, F., Long, D., Sotta, P., 2002. Evidence for the shift of the glass transition near the particles in silica-filled elastomers. Macromolecules 35, 9756-9762.

Berriot, J., Montes, H., Lequeux, F., Long, D., Sotta, P., 2003. Gradient of glass transition temperature in filled elastomers. Europhys. Lett. 64 50-56.

Brunac, J.B., Gerardin, O., Leblond, J.B., 2009. On the heuristic extension of Haigh's diagram for the fatigue of elastomers to arbitrary loadings. Int. J. Fatigue 31 (5), 859-867.

Celina, M., Wise, J., Ottesen, D.K., Gillen, K.T., Clough, R.L., 1998. Oxidation profiles of thermally aged nitrile rubber. Polymer Degrad. Stability 60, 493-504.

Delor-Jestina, F., Lacoste, J., Barrois-Oudin, N., Cardinet, C., Lemaire, J., 2000. Photo-thermal natural ageing of ethylene-propylene-diene monomer (epdm) rubber used in automotive applications influence of carbon black crosslinking and stabilizing agents. Polymer Degrad. Stability 67, 469-477.

Diani, J., Brieu, M., Vacherand, J.-M., Rezgui, A., 2004. Directional model for isotropic and anisotropic hyperelastic rubber-like materials. Mech. Mater. 36 (4), 313-321.

Einstein, A., 1906. Zur theorie der bownschen bewegung. Ann. Phys. 17, 549 .

Feller, R.L., 1994. Accelerated aging, photochemical and thermal aspects. The Getty Conservation Institute.

Fletcher, W.P., Gent, A.N., 1953. Non-linearity in the dynamic properties of vulcanised rubber compounds. Trans. Inst. Rubber. Ind. 29, 266280

Kumar, A., Commereuc, S., Verney, V., 2004. Ageing of elastomers: a molecular approach based on rheological characterization. Polymer Degrad. Stability 85 (2), 751-757.
Le Cam, J.-B., Toussaint, E., 2009. Cyclic volume changes in rubbers. Mech. Mater. 41, 898-901.

Litvinov, V.M., Steeman, P.A.M., 1999. Epdm-carbon black interactions the reinforcement mechanisms as studied by low-resolution h-1 nmr. Macromolecules 32 (25), 8476-8490.

Machado, G., Chagnon, G., Favier, D., 2010. Analysis of the isotropic models of the Mullins effect based on filled silicone rubber experimental results. Mech. Mater. 42 (9), 841-851.

Maier, P.G., Goritz, D., 1996. Molecular interpretation of the Payne effect. Kaut. Gummi Kunstst. 49 (1), 18-21.

Medalia, A.I., 1978. Effect of carbon black on dynamic properties of rubber vulcanizates. Rubber Chem. Technol. 51 (3), 437-523.

Merabia, S., Sotta, P., Long, D., 2008. Macromolecules 41, 8252-8266.

Morozov, I., Lauke, B., Heinrich, G., 2010. A new structural model of carbon black framework in rubbers. Comput. Mater. Sci. 47 (3), 817-825.

Palmieri, G., Sasso, M., Chiappini, G., Amodio, D., 2009. Mullins effect characterization of elastomers by multi-axial cyclic tests and optical experimental methods. Mech. Mater. 41, 1059-1067.

Payne, A.R., 1962. The dynamic properties of carbon black-loaded natural rubber vulcanizates. Part I. J. Appl. Phys. 6 (19), 57-63.

Rendek, M., Lion, A, 2010. Strain induced transient effects of filler reinforced elastomers with respect to the Payne-effect: experiments and constitutive modelling. ZAMM 90 (5), 436-458.

Suzuki, N., Ito Masayoshi, M., Ono, S., 2005. Effects of rubber/filler interactions on the structural development and mechanical properties of nbr/silica composites. J. Appl. Polym. Sci. 95 (1), 74-81.

Voet A., 1975. Dynamic mechanical properties and electrical resistance of carbon black filled vulcanized at minor and major extensions. In: Colloq Int CNRS Obernai.

Wang, M.J., 1999. The role of filler networking in dynamic properties of filled rubber. Rubber Chem. Technol. 72 (2), 430-448.

Wang, M.J., Wolff, S., Donnet, J.B., 1991a. Filler elastomers interactions 1 silica surface energies and interactions with model compounds. Rubber Chem. Technol. 64 (4), 559-576.

Wang, M.J., Wolff, S., Donnet, J.B., 1991b. Filler elastomers interactions 3 carbon-black-surface energies and interactions with elastomers analogs. Rubber Chem. Technol. 64 (5), 714-736. 